\title{
Collective choice under dichotomous preferences
}

\author{
Anna Bogomolnaia, \\ Hervé Moulin and Richard Stong
}

\author{
Rice University
}

revised, August 2003

\begin{abstract}
Agents partition deterministic outcomes into good or bad. A direct revelation mechanism selects a lottery over outcomes - also interpreted as time-shares. Under such dichotomous preferences, the probability that the lottery outcome be a good one is a canonical utility representation.

The utilitarian mechanism averages over all deterministic outcomes "approved" by the largest number of agents. It is efficient, strategyproof and treats equally agents and outcomes.

We reach the impossibility frontier if we also place the lower bound $\frac{1}{n}$ on each agent's utility, where $n$ is the number of agents; or if this lower bound is the fraction of good outcomes to feasible outcomes.

We conjecture that no ex-ante efficient and strategyproof mechanism guarantees a strictly positive utility to all agents at all profiles, and prove a weaker version of this conjecture.
\end{abstract}


Keywords: collective choice, dichotomous preferences, randomization, time-sharing, strategyproofness, fair share.

Hervé Moulin and Anna Bogomolnaia, Department of Economics, MS 22, Rice University, P.O. Box 1892, Houston, Texas 77251-1892, USA, moulin@rice.edu, http://www.ruf.rice.edu/ ${ }^{\sim}$ econ/papers/index.html

Richard Stong, Department of Mathematics, MS 136, Rice University, P.O. Box 1892, Houston, Texas 77251-1892, USA, stong @ rice.edu.

\section{JEL: D71, C78, D63}

Acknowledgements: Stimulating conversations with Salvador Barberà, Eric Friedman, François Maniquet, Ron Holzman, Scott Shenker, Yves Sprumont, Rakesh Vohra were very helpful. The critical comments of the associate editor and a referee are gratefully acknowledged. The referee in particular suggested the statement of Proposition 4 .

Moulin's research is supported by the NSF under grant SES0112032. 


\section{Collective choice under dichotomous preferences}

\section{Introduction and overview of the results}

Randomization is surely the oldest and most practical tool to achieve equity in collective decision making. A lottery chooses one of several mutually exclusive outcomes: the weights of the lottery (the probability distribution over the deterministic outcomes) are adjusted to achieve a "fair" compromise. Say that we have three outcomes $a, b, c$, and ten concerned agents split into 2 supporters of $a, 5$ of $b$ and 3 of $c$ : a prima facie fair compromise is the lottery $p_{a}=.2, p_{b}=.5$, $p_{c}=.3$.

An alternative interpretation of the convex combination of outcomes is timesharing. Think of our agents sharing a "source" such as a TV or radio in a public space (a gym, the living room of the house they share), and allocating timeshares to the available channels.

In this paper we revisit the classic negative result on collective choice using randomization/time-sharing under the assumption that the preferences are dichotomous. That is, each agent sorts the outcomes as either "good" or "bad" (possibly "all good" or "all bad"); her preferences over deterministic outcomes have at most two indifference classes.

This is a considerable restriction of the standard domain of rational preferences (complete and transitive). Yet dichotomous preferences arise naturally in a number of time-sharing problems. In the TV example, the channels may broadcast the same news program in a variety of languages, and each agent understands a certain subset of these languages. Or think of an antenna broadcasting to geographically dispersed agents; for each orientation of the antenna, only a certain subset of agents receive an adequate signal, hence their preferences over the feasible set of orientations are dichotomous. ${ }^{1}$ Or the source may be choosing the software to communicate with a number of machines, and each machine is only endowed with some of the softwares available to the source.

Scheduling problems provide another natural example where preferences are dichotomous. We must schedule a meeting at one of several possible times, and each potential participant can only attend at a certain subset of those times. Everyone wishes to maximize the probability that he can attend the meeting. ${ }^{2}$

We investigate the compatibility, in the dichotomous domain, of the three perennial goals of mechanism design: efficiency, incentive compatibility and fairness.

Incentive compatibility takes the standard form of strategyproofness: truthtelling is a dominant strategy in the direct mechanism where agents report their preferences.

\footnotetext{
${ }^{1}$ This example was suggested by Scott Shenker.

${ }^{2}$ We thank the Associate Editor for suggesting this example.
} 
Efficiency is the usual Pareto optimality of the lottery/time-sharing. In the lottery interpretation, a weaker property is also of interest: it only requires that each deterministic outcome chosen with positive probability be Pareto optimal. To distinguish these two properties, we speak of ex-post efficiency for the latter, and of ex-ante efficiency for the former. We stress that ex-post efficiency has no convincing interpretation in the time-sharing context.

The familiar notion of fairness known as horizontal equity is captured by the two properties of anonymity and neutrality, expressing the symmetric treatment of, respectively, agents and outcomes. Our first observation - Proposition 1 in Section 4.1 - is a positive result. Under dichotomous preferences, the four requirements of ex-ante efficiency, strategyproofness, anonymity and neutrality are compatible. A simple example is the mechanism we call utilitarian, reminiscent of approval voting; that randomizes uniformly over all outcomes with the largest "approval" (i.e., outcomes viewed as "good" for the greatest number of agents).

Two normative shortcomings of the utilitarian mechanism are that it may be vulnerable to misrepresentation by groups (coalitions) of agents, and it exhibits the classic "tyranny of the majority." We take these two criticisms in turn.

Consider a situation with three agents $A, B, C$ and three outcomes $a, b, c$ where for agent $X$, outcome $x$ is the only good one. The utilitarian mechanism chooses each outcome with probability $\frac{1}{3}$. However, if both agents $A$ and $B$ pretend that they like both $a$ and $b$ - and dislike $c$ - whereas agent $C$ remains truthful, the utilitarian lottery picks $a$ and $b$ each with probability $\frac{1}{2}$, and the joint misreport by $A$ and $B$ benefits them both. Propositions 2 and 3 in Section 4.2 generalize this observation: they show that the requirement of groupstrategyproofness is simply too demanding in our problem: it is not even compatible with ex-post efficiency.

The second objection to the utilitarian mechanism is more serious. An agent who dislikes all utilitarian outcomes (i.e., all outcomes with largest approval) gets no share at all of the collective benefits, his own preferences are entirely ignored. To avoid this "tyranny of the majority," we can impose one of two natural lower bounds on individual benefits.

The first lower bound, that we call fair outcome share, views the uniform lottery over all outcomes as a disagreement option that each agent has the right to enforce. This rewards agents who like a relatively large subset of feasible outcomes: if $q$ among the $p$ outcomes are good for me, the probability that one of my good outcomes is eventually selected is at least $\frac{q}{p}$.

The second lower bound, called fair welfare share uses the random dictator mechanism as the disagreement option that each participant is entitled to enforce. In other words, we give a fair share of control over the final outcome to each participant. Formally, this lottery guarantees, for each one of the $n$ agents, that one of her good outcomes is chosen with probability at least $\frac{1}{n}$. These two bounds are obviously incompatible: suppose $n=2$ and Ann likes only outcomes $a, b$ whereas Bob only likes $c$; we can't simultaneously choose one of $a, b$ with probability at least $\frac{2}{3}$, and $c$ with probability at least $\frac{1}{2}$. 
Suppose $^{3}$ two colleagues have lunch together every day of the week at one of five feasible restaurants, serving respectively Indian, Greek, Italian, French and Chinese food. Ann strongly prefers Indian food, and finds the other four equally dull, whereas Bob is equally happy in all but the Indian restaurant, where the spicy food does not appeal to him. The fair outcome share suggests to visit the Indian restaurant exactly one day per week, a reasonable compromise given that preferences reflect tastes. But suppose instead that preferences reflect needs: Ann is religiously vegetarian, and the Indian place is the only one offering a vegetarian menu; Bob is allergic to Indian spices. Then the fair welfare share makes more sense: it suggests that our two colleagues should visit the Indian restaurant every other day (and every day one of them will bring a lunch bag ).

Our main results describe the compatibility, or lack thereof, of efficiency and incentive-compatibility with either one of the two lower bounds. Proposition 4 in Section 5.2 shows that the fair outcome share is not feasible for any strategyproof and ex-post efficient mechanism. By contrast, it is easy to design a strategyproof and ex-post efficient mechanism guaranteeing the fair welfare share (and horizontal equity as well): Proposition 5 in Section 5.3. A simple example obtains by adapting the familiar random dictator mechanism in the presence of indifferences: the random priority mechanism selects with uniform probability an ordering of the agents, then maximizes lexicographically their preferences. See Section 5.3 for details.

Theorem 1 in Section 5.4 reveals an important tradeoff among strategyproof mechanisms between the fair welfare share and ex-ante efficiency. It shows that no strategyproof and ex-ante efficient mechanism can meet the fair welfare share and be horizontally equitable. We suspect that horizontal equity is in fact redundant in this statement.

A considerably weaker version of the lower bound on individual utilities is sufficient to rule out the tyranny of the majority. The property that we call positive shares merely requires that for any participant, the probability that one of his good outcomes be selected is strictly positive. In other words, every one gets some share of the collective benefits. We submit as a challenging conjecture the following statement: there is no strategyproof and ex-ante efficient mechanism guaranteeing positive shares.

We have been able to prove a weaker version of this statement, where strategyproofness is strengthened into preference monotonicity. Consider a shift in preferences where the status of a certain outcome for a certain agent goes from bad to good: preference monotonicity requires that the probability of each and every other outcome should not increase. This property is akin to gross substitutability in demand theory. Theorem 2 in Section 6 shows the incompatibility of ex-ante efficiency, preference monotonicity and positive shares.

We relate our work to the literature in the next section. The model is defined in Section 3, and our first results on strategyproofness and groupstrategyproofness are in Section 4. The two lower bounds on individual welfares are the subject of Section 5, whereas positive shares is discussed in Section 6 .

\footnotetext{
${ }^{3}$ We thank an anonymous referee for suggesting this example.
} 
Section 7 briefly discusses a natural family of ex-ante efficient solutions. All non-trivial proofs are gathered in the Appendix.

\section{Relation to the literature}

Recall first the central "negative" result of the literature on collective choice with lotteries/time-sharing in the standard preference domain, i.e., when agents have complete and transitive preferences over deterministic outcomes, and Von Neumann-Morgenstern utilities over lotteries.

Consider the random dictator mechanism: an agent is randomly selected, with equal probability for each agent, and chooses freely the final outcome. This mechanism is horizontally equitable and strategyproof (we ignore tie-breaking issues for brevity). It is also ex-post efficient, namely every (deterministic) outcome selected with a positive probability is Pareto optimal. However, it may not be ex-ante efficient: the resulting lottery over outcomes may be Pareto inferior. In the example with 10 agents at the beginning of Section 1, imagine a fourth outcome $d$ that is a good compromise between $a, b$, and $c$. For instance, VNM utilities are:

$\begin{array}{lllll}\text { \# of agents } & u(a) & u(b) & u(c) & u(d) \\ 2 & 11 & 0 & 0 & 10 \\ 5 & 0 & 11 & 0 & 10 \\ 3 & 0 & 0 & 11 & 10\end{array}$

The utility of the random priority lottery is $2.2,5.5$, and 3.3 for the three types of agents respectively, well below the 10 utils from choosing $d$.

Conversely, the random dictator mechanism is essentially characterized by anonymity (equal treatment of agents), strategyproofness, and ex-post efficiency $^{4}, 5$. But an ex-ante efficient and strategyproof mechanism must involve a full time dictator, namely an agent such that, for any utility profile, any outcome chosen with positive probability is among the dictator's top outcomes. These results originate in Gibbard [1977], the seminal paper on collective choice with lotteries as outcomes, that however restricts attention to mechanisms eliciting only ordinal preferences over deterministic outcomes; the same is true of Barbera [1979], who describes the fair and strategyproof - yet inefficient mechanisms in this class. The tiny literature on mechanisms eliciting complete Von Neumann-Morgenstern utilities consists of Gibbard [1978], Hylland [1980], Freixas [1984], Barbera, Bogomolnaia and Van der Stel [1998], and Nandeibam [2001].

When preferences are dichotomous over deterministic outcomes, first order stochastic dominance is a complete preference ordering of the lotteries. A canonical utility representation is the probability that a given lottery assigns to the

\footnotetext{
${ }^{4}$ Nandeibam [2001] shows that a much weaker form of efficiency is sufficient for the result: when a certain outcome is the top for every agent, it must be chosen with probability one. Moreover, if we drop the anonymity requirement, the only new mechanisms choose a dictator according to an arbitrary fixed probability distribution.

${ }^{5}$ When preferences over lotteries (or any other convex set of outcomes) are strictly convex, a similar characterization result obtains: Dutta, Peters and Sen [1999].
} 
set of good outcomes. Thus the VNM axiomatic construction is pointless in this preference domain, and the mechanisms eliciting ordinal preferences are the only ones we need to consider.

In our model the random dictator idea leads to the random priority mechanism, that remains a fair, strategyproof and ex-post efficient mechanism (see Section 5.3); however, these properties no longer characterize the mechanism. Another new fact in the dichotomous domain is the compatibility of ex-ante efficiency, strategyproofness and horizontal equity; as explained above, these three properties are incompatible in the classic domain.

The companion piece Bogomolnaia and Moulin [2001] develops a related model of random assignment, under the same assumptions of dichotomous preferences. The problem is to assign to each agent at most one from a set of heterogeneous objects, when each agent partitions the objects as "good" or "bad". Randomization (time-sharing) restores fairness. The main simplification of the assignment problem, relative to the general decision problem discussed here, is that a random assignment is efficient if and only if it is utilitarian (maximizes the sum of utilities); in particular ex-post and ex-ante efficiency coincide. It is then easy to find an efficient and strategyproof mechanism guaranteeing a "fair" share to every participant: the random priority mechanism is an example, the revelation mechanism selecting the Nash solution is another. Moreover the latter mechanism is group-strategyproof as well.

\section{The model}

The set $N$ of agents is finite, and so is the set $A$ of (deterministic) outcomes. These two sets are fixed throughout.

A dichotomous preference on $A$ is described by a row vector $v \in\{0,1\}^{A}$ with the interpretation that outcome $a$ is good if $v^{a}=1$ and bad if $v^{a}=0$. We also say that an agent likes $a$ if $v^{a}=1$ and dislikes it if $v^{a}=0$. Note that $v^{a}=0$ for all $a$, and $v^{a}=1$ for all $a$, represent the same preference: agents who are thus indifferent will not matter in any of the mechanisms discussed below.

A $N$-profile of dichotomous preferences is a $N \times A$ matrix $U$ with entry $u_{i}^{a}=0$ or $1: U \in\{0,1\}^{N \times A}$. The $i$-row $U_{i}$ is agent $i$ 's preference, and the $a$ column $U^{a}$ is the set of agents who like outcome $a$. Abusing notations slightly, we also use $U_{i}$ to represent the set of outcomes that agent $i$ likes.

A problem is a triple $(N, A, U)$ and a lottery (vector of time-shares) is a column vector $p$ in the $A$-simplex: $p_{a} \geq 0$ for all $a$ and $\sum_{A} p_{a}=1$. The canonical utility of agent $i$ for the lottery $p$ is thus written as $u_{i}(p)=U_{i} \bullet p=\sum_{A} u_{i}^{a} \cdot p_{a}$.

A solution to the problem $(N, A, U)$ is a lottery $p$ deemed desirable according to certain properties of efficiency and fairness (defined below). When we discuss properties relating the solutions of different problems, we speak of a mechanism.

Given $N$ and $A$, a mechanism is a mapping $\pi$ selecting for each problem $(N, A, U)$ a solution $p=\pi(U)$. The following three properties of a mechanism are standard, and require no further comments:

Anonymity: for any profile $U$, and permutation $\sigma$ of $N$ 
$\pi(U)=\pi\left({ }^{\sigma} U\right)$ where ${ }^{\sigma} U_{i}=U_{\sigma(i)}$

Neutrality: for any profile $U$, and permutation $\tau$ of $A$

$\pi\left({ }^{\tau} U\right)={ }^{\tau} \pi(U)$ where ${ }^{\tau} p_{a}=p_{\tau(a)}$ and ${ }^{\tau} U^{a}=U^{\tau(a)}$

Strategyproofness (SP): for any $i \in N$, and profiles $U, U^{\prime}$

$\left\{U_{j}=U_{j}^{\prime}\right.$ for all $\left.j \neq i\right\} \Rightarrow\left\{U_{i} \bullet \pi(U) \geq U_{i} \bullet \pi\left(U^{\prime}\right)\right\}$

An outcome $a, a \in A$, is efficient at profile $U$ if, for every other outcome $b$ $\left\{U^{a} \leq U^{b}\right\} \Rightarrow\left\{U^{a}=U^{b}\right\}$. We distinguish two notions of efficiency for a lottery $p$ :

Ex-Post Efficiency (EXP): for all $a$ in $A\left\{p_{a}>0\right\} \Rightarrow\{a$ is efficient $\}$

Ex-Ante Efficiency (EXA): for all lotteries $p^{\prime}\left\{U \bullet p \leq U \bullet p^{\prime}\right\} \Rightarrow\{U \bullet p=$ $\left.U \bullet p^{\prime}\right\}$

Ex-ante efficiency coincides with ex-post efficiency for a deterministic outcome $a$, but for lotteries the latter is a strictly weaker requirement than the former. The simplest example involves five outcomes and six agents: see Figure 1. All five outcomes are efficient, thus any lottery is ex-post efficient. However $p, p_{c}=p_{d}=p_{e}=\frac{1}{3}$, is strictly Pareto inferior to $q, q_{a}=q_{b}=\frac{1}{2}$.

\section{Strategyproofness and groupstrategyproofness}

\subsection{The utilitarian solution}

Our first result shows that ex-ante efficiency, strategyproofness and horizontal equity are compatible in our model. Its obvious proof is omitted. Given a problem $(N, A, U)$ we denote by $A_{1}$ the subset of outcomes liked by the largest number of agents:

$$
a \in A_{1} \Leftrightarrow\left|U^{a}\right| \geq\left|U^{b}\right| \text { for all } b \in A
$$

Definition 1 The utilitarian solution for problem $(N, A, U)$ is the uniform lottery $p^{u}$ over $A_{1}$ :

$$
p_{a}^{u}=\frac{1}{\left|A_{1}\right|} \text { if } a \in A_{1} ; p_{a}^{u}=0 \text { otherwise }
$$

Proposition 1 The utilitarian mechanism is anonymous, neutral, strategyproof and ex-ante efficient.

We note that the four properties listed in Proposition 1 are by no means sufficient to characterize the utilitarian mechanism.

Consider first the simple variant of the utilitarian solution in which two outcomes $a, a^{\prime}$ in $A_{1}$ with identical support are conventionally identified. Given $(N, A, U)$, define the equivalence relation $\sim$ on $A: a \sim a^{\prime} \Leftrightarrow U^{a}=U^{a^{\prime}}$, and choose a single outcome $a^{*}$ in each equivalence class. The new solution is the uniform lottery over the set of all outcomes $a^{*}$ : the corresponding utility profile 
is unique (if the lottery itself is not) and generally differ from the utilitarian profile if the indifference classes are of different sizes. It is easy to check that the corresponding direct revelation mechanism(s) meets the four properties in Proposition 1.

Our next example is a solution that gives positive weight to non-utilitarian outcomes at many profiles. This solution gives most of the weight to utilitarian outcomes, who are each liked by exactly $m$ agents; it gives also a smaller weight to an outcome $a$ liked by exactly $m-1$ agents if none of the agents who like $a$ also likes some utilitarian outcome. If there are no such outcomes, our solution coincides with the utilitarian one.

We fix $(N, A)$ and we choose a positive weight $w$ such that $w \geq|A|-1$. Given a problem $(N, A, U)$, we define $m=\max _{A}\left|U^{a}\right|$, the maximal support, $A_{1}=\arg \max _{A}\left|U^{a}\right|$ the set of utilitarian outcomes, and

$$
N_{1}=\cup_{A_{1}} U^{a} ; B=\left\{a \in A:\left|U^{a}\right|=m-1 \text { and } U^{a} \cap N_{1}=\varnothing\right\} .
$$

The lottery $p$ is now

$$
p_{a}=\frac{w}{\left|A_{1}\right| w+|B|} \text { if } a \in A_{1} ; p_{a}=\frac{1}{\left|A_{1}\right| w+|B|} \text { if } a \in B ; p_{a}=0 \text { otherwise. }
$$

Lemma 1 The solution $p$ defines an anonymous, neutral, strategyproof and exante efficient mechanism.

\subsection{Groupstrategyproofness: impossibility results}

The utilitarian mechanism is robust against strategic misreporting by a single agent, but is eminently vulnerable to joint misreporting by several agents. Recall the three agents/three outcomes example given in Section 1. We show in this subsection that all efficient and horizontally equitable mechanisms are similarly manipulable.

The familiar strengthening of strategyproofness to coalitions writes as follows for an arbitrary mechanism $\pi$ :

Group-Strategyproofness (GSP): for all $S \subseteq N$, and profiles $U, U^{\prime}$

$\left\{U_{j}=U_{j}^{\prime}\right.$ for all $j \in N \backslash S$, and $U_{i} \bullet \pi\left(\overline{U^{\prime}}\right) \geq U_{i} \bullet \pi(U)$ for all $\left.i \in S\right\} \Rightarrow$ $U_{i} \bullet \pi\left(U^{\prime}\right)=U_{i} \bullet \pi(U)$ for all $i \in S$.

The next result shows that this property is not even compatible with the weak version of efficiency.

Proposition 2 Assume $|N| \geq 3$ and $|A| \geq 3$. No mechanism is both ex-post efficient and group-strategyproof.

A weaker requirement than GSP only rules out misreporting that strictly improves the utility of each member of the deviating coalition.

Weak Group-Strategyproofness for all $S \subseteq N$, and profiles $U, U^{\prime}$ :

$\left\{U_{j}=U_{j}^{\prime}\right.$ for all $\left.j \in N \backslash S\right\} \Rightarrow\left\{U_{i} \bullet \pi\left(U^{\prime}\right) \leq U_{i} \bullet \pi(U)\right.$ for some $\left.i \in S\right\}$

Weak Group-Strategyproofness is not incompatible with efficiency, even in the ex-ante sense. To prove this claim, we introduce a family of mechanisms playing an important role in the next section as well. 
Definition 2 Fix an arbitrary ordering $\sigma$ of $N$, say $i_{1}>i_{2}>\ldots>i_{n}$, and a preference profile $U$. Say that outcome $a$ is a $\sigma$-priority outcome at $U$ if $U^{a}$ is a lexicographic maximum for the ordering $\sigma$ : for any $b \in A$, there exists an integer $m, 0 \leq m \leq n$, such that:

$u_{i_{k}}^{a}=u_{i_{k}}^{b}$ for $k=1, \ldots, m$ and $u_{i_{k+1}}^{a}>u_{i_{k+1}}^{b}$ (where $m=0$ if $u_{i_{1}}^{a}>u_{i_{1}}^{b}$ and $m=n$ if $\left.U^{a}=U^{b}\right)$.

A mechanism $\pi$ is a $\sigma$-priority mechanism if it selects at every profile $U$ a lottery over the set of $\sigma$-priority outcomes.

Notice that, for a given ordering $\sigma$, the utility profile of all $\sigma$-priority mechanisms is the same; abusing language, we speak of the $\sigma$-priority direct revelation mechanism. This mechanism is obviously weakly groupstrategyproof and ex-ante efficient. However, the average over $\sigma$ of all such mechanisms (called random priority mechanism in the next section: see Definition 3 in Section 5.3) is not weakly groupstrategyproof. This is an instance of a general impossibility result.

Proposition 3 Assume $|N| \geq 4$ and $|A| \geq 6$. An anonymous and neutral mechanism cannot be both ex-post efficient and weakly group-strategyproof.

Proposition 3 is a tight statement. A $\sigma$-priority mechanism shows that we cannot drop anonymity. The following non neutral utilitarian mechanism shows that we cannot drop neutrality either. Fix an arbitrary ordering of $A$ and select - with probability one - the smallest outcome in $A_{1}$ according to this ordering: this mechanism is efficient ex-ante, weakly groupstrategyproof and anonymous.

\section{Fair share}

\subsection{Two definitions of fair share}

We define formally the two individual rationality properties discussed in Section 1. Given a problem $(N, A, U)$ we write $p^{*}$ for the uniform lottery on $A$, and we consider two properties of a solution $p$ :

Fair Outcome Share (FOS): for all $i \in N\left\{U_{i} \neq 0\right\} \Longrightarrow U_{i} \bullet p \geq U_{i} \bullet p^{*}$

Fair Welfare Share (FWS): for all $i \in N\left\{U_{i} \neq 0\right\} \Longrightarrow U_{i} \bullet p \geq \frac{1}{|N|}$

The fair outcome share rewards agents for whom more outcomes are good: the lower bound on an agent utility is precisely the proportion of good outcomes in his/her preferences. The fair welfare share, on the other hand, places the same lower bound on the utility of all agents who are not completely indifferent over $A$. In the terminology of bargaining theory, the disagreement point is $p^{*}$ under FOS and is the random priority mechanism under FWS (Definition 3 below). Recall that these two properties are mutually incompatible at many preference profiles if we have at least two agents and three outcomes. 


\subsection{Fair outcome share: a simple impossibility result}

Consider the three agents/three outcomes problem in Figure 2: $U_{1}=\{a\}, U_{2}=$ $\{b\}, U_{3}=\{a, b\}$. Suppose we are given a mechanism $\pi$ that is ex-post efficient and meets FOS. Let $p$ be the lottery selected by $\pi$ at this profile. Assume first $p_{a}<\frac{2}{3}$. If agent 1 reports $U_{1}^{\prime}=\{a, c\}$ the lottery $p^{\prime}=\pi\left(U_{1}^{\prime}, U_{2}, U_{3}\right)$ has $p_{c}^{\prime}=0$ (by ex-post efficiency) and $p_{a}^{\prime}+p_{c}^{\prime} \geq \frac{2}{3}$ by (FOS). Therefore $U_{1} \bullet p^{\prime} \geq \frac{2}{3}>U_{1} \bullet p$, and agent 1's misreport is a profitable manipulation. If $p_{a} \geq \frac{2}{3}$, then $p_{b}<\frac{2}{3}$ and agent 2 can similarly manipulate. We have proven

Proposition 4 Assume $|N| \geq 3$ and $|A| \geq 3$. No mechanism is ex-post effcient, strategyproof, and meets the fair outcome share.

Proposition 4 is a tight statement. We omit the obvious proof.

Remark 1 Consider the weaker variant of the FOS requiring only $U_{i} \bullet p \geq$ $\varepsilon \cdot\left(U_{i} \bullet p^{*}\right)$ for some fixed parameter $\varepsilon, 0<\varepsilon<1$. Although much weaker than the FOS when $\varepsilon$ is very small, when $\min \{|N|,|A|\}$ is large enough this property cannot be met by an ex-post efficient and strategyproof mechanism. The proof uses the following profile with $m+1$ agents and $m+l$ outcomes $a_{1}, . ., a_{m}, b_{1}, \ldots b_{l}: U_{0}=\left\{a_{1}, \ldots, a_{m}\right\} ; U_{i}=\left\{a_{i}\right\}$ for $i=1, \ldots, m$. When agent $i$ reports $U_{i}^{\prime}=\left\{a_{i}, b_{1}, \ldots, b_{l}\right\}$, ex-post efficiency and FOS imply that the corresponding lottery $p^{\prime}$ must select $a_{i}$ with probability at least $\frac{l+1}{m+l}$. When $l, m$ are large enough, this brings a contradiction of strategyproofness.

\subsection{The random priority mechanism}

In sharp contrast to the incompatibility stated in Proposition 4, the fair welfare share is satisfied by some strategyproof and ex-post efficient mechanisms. The simplest example is the following mechanism.

Definition 3 Given $(N, A, U)$ a mechanism $\pi$ is a random priority solution if there exists, for all ordering $\sigma$ of $N$, a $\sigma$-priority mechanism $\pi^{\sigma}$ such that:

$$
\pi=\frac{1}{|N| !} \sum_{\sigma} \pi^{\sigma}
$$

All such mechanisms yield the same utility profile at all preference profiles.

Proposition 5 The random priority mechanism is anonymous, neutral and meets the fair welfare share; it is strategyproof and ex-post efficient. It is not ex-ante efficient.

Anonymity, neutrality and the fair welfare share are obvious. For any fixed ordering $\sigma$, the $\sigma$-priority mechanism is strategyproof, and this property is pre-

served by convex combinations with fixed coefficients. Finally the example of Figure 1 shows a failure of ex-ante efficiency. Here the $\sigma$-priority outcome is $c$ 
if $\sigma$ starts with agent 1 and agent 4 has a higher priority than 2 and 3 , or if $\sigma$ starts with 4 and agent 1 is higher than 5 and 6 . Thus $p_{c}=p_{d}=p_{e}=\frac{1}{9}$, $p_{a}=p_{b}=\frac{1}{3}$, and $u_{i}=\frac{4}{9}$ for all $i$, whereas $q_{a}=q_{b}=\frac{1}{2}$ yields $u_{i}=\frac{1}{2}$ for all $i$.

It is easy to generalize this example so that the random priority lottery wastes an arbitrary large fraction of total surplus.

We note that the random priority mechanism is not the only one satisfying the properties listed in Proposition 5. We construct another example as follows. Say that outcome $a$ is $i$-utilitarian if $a \in U_{i}$ and $\left|U^{a}\right| \geq\left|U^{b}\right|$ for all $b \in U_{i}$. If $i$ is indifferent between all outcomes, this is just the definition of a utilitarian outcome. Define the $i$-utilitarian solution as the uniform lottery over all $i$-utilitarian outcomes, and the $*$-utilitarian solution as the average of the $i$-utilitarian solutions over all $i$.

We conclude this subsection with a natural family of scheduling problems with dichotomous preferences where the difference between ex-ante and ex-post efficiency is typically small, reinforcing the appeal of the random priority solution.

Example 1 A server is endowed with $T$ units of resources. Serving agent $i$ requires $x_{i}$ units of resources. To fix ideas, think of $x_{i}$ as the time needed to complete job $i$, and the server is alive for exactly $T$ periods. Agent $i$ 's utility is 1 if her job is completed within these $T$ periods, 0 otherwise (incomplete jobs bring zero utility).

A vector $a \in\{0,1\}^{N}$ is a feasible (deterministic) utility vector if and only if $x \bullet a \leq T$ where $x=\left(x_{i}\right)$. By efficiency we only need to consider the maximal feasible vectors a, i.e., those for which the set of satisfied agents is inclusionmaximal. Denote by $A$ the set of these vectors, and simply take the column vector a to be the corresponding column of $U$. All outcomes of $A$ are efficient by construction, but a lottery over A may well be ex-ante inefficient.

However, suppose that all outcomes a exhaust the $T$ periods: $x \bullet a=T$ for all $a$. This holds true for instance if there are at least $T$ agents with a 1-period job. Then every feasible utility vector u (every convex combination of the column vectors a) has $x \bullet u=T$ as well, hence is ex-ante efficient: the two notions of efficiency coincide . $^{6}$

Another interesting case is when the length of the different jobs are not too different. Then the efficiency loss incurred at any (ex-post efficient) lottery will be small ${ }^{7}$.

\subsection{Fair welfare share: an impossibility result}

Theorem 2 Assume $|N| \geq 5$ and $|A| \geq 17$. An anonymous and neutral mechanism cannot be ex-ante efficient, strategyproof, and meet the fair welfare share.

\footnotetext{
${ }^{6}$ Conversely, every preference profile $U$ for which the two notions of efficiency coincide can be obtained in this way ( $\mathrm{X}$ and Holzman [2003]).

${ }^{7}$ Set $\alpha=\max _{i} x_{i}$ and $\beta=\min _{i} x_{i}$. Efficiency of the deterministic outcome $a$ implies $T<x \bullet a+\alpha$ (ignoring the trivial case where all agents can be served at the same time). Therefore $T<x \bullet u+\alpha$ for any feasible utility vector. If $u^{\prime}$ is feasible and Pareto superior to $u$, inequalities $x \bullet u^{\prime} \leq T<x \bullet u+\alpha$ imply easily $\sum_{i} u_{i}^{\prime}-\sum_{i} u_{i}<\frac{\alpha}{\beta}$.
} 
In the above statement, we cannot dispense with either one of ex-ante efficiency, strategyproofness, or fair welfare share. The random priority mechanism meets all properties but ex-ante efficiency. The utilitarian one meets all but the fair welfare share. Finally the Nash solution (maximizing the product of utilities $U_{i} \bullet p$ ) defines a mechanism meeting all properties but strategyproofness: see the discussion of this solution in Section 7.

However, we have not been able to determine if one of the anonymity or neutrality property (or both) can be dropped.

\section{Positive shares and more impossibility}

In Section 4 we presented a few ex-ante efficient and strategyproof mechanisms: see Proposition 1 and the discussion following it. All of them violate our two "fair share" lower bounds, and, more radically, they give no benefit at all to some agents in most problems. To rule out such "tyranny of the majority" the following very weak form of individual rationality is enough:

$$
\text { Positive Shares: for all } i \in N\left\{U_{i} \neq 0\right\} \Longrightarrow\left\{U_{i} \bullet p>0\right\}
$$

Is there any ex-ante efficient and strategyproof mechanism meeting Positive Shares as well? This is a challenging open question to which we suspect the answer is negative when $|N|$ and $|A|$ are large enough.

Our intuition for this conjecture is based on a logically weaker result stated below as Theorem 3. There we strengthen strategyproofness into a natural monotonicity property reminiscent of the gross substitutability theory in demand theory. The property is defined for a given pair $N, A$ and a mechanism $\pi$ :

Preference Monotonicity (PM): for any profiles $U, V$ and any outcome $a$ : $\left\{u_{i}^{a} \leq v_{i}^{a}\right.$ and $u_{i}^{b}=v_{i}^{b}$ for all $i$, all $\left.b \neq a\right\} \Longrightarrow\left\{\pi_{b}(U) \geq \pi_{b}(V)\right.$ for all $\left.b \neq a\right\}$

Whenever a certain outcome $a$ becomes more popular (in the sense that one or more agents for whom $a$ was bad, now see it as good) the probability/timeshare of every other outcome cannot increase. It is easy to check that the utilitarian and random priority mechanisms are both preference monotonic.

Preference Monotonicity is interesting in its own right: it rules out crosssubsidization between outcomes (lobbying to increase the support of $a$ cannot increase the time-share of $b$ ).

Lemma 2 Preference Monotonicity implies strategyproofness.

Proof. We consider the simple case where agent $i$ with true utility $U_{i}$, reports instead $U_{i}^{\prime}$ where $U_{i}^{\prime} \backslash U_{i}=\{a\}$ and $U_{i} \backslash U_{i}^{\prime}=\{b\}$. The general case is just as easy. Set $U_{i}^{\prime \prime}=U_{i} \backslash\{b\}$ and write $U^{\prime}, U^{\prime \prime}$ for the profiles where $U_{i}$ 
is replaced by $U_{i}^{\prime}$ and $U_{i}^{\prime \prime}$ respectively. By $P M$ and $U_{i}^{\prime}=U_{i}^{\prime \prime} \cup\{a\}$ we have successively:

$$
\begin{aligned}
\pi_{x}(U) & \leq \pi_{x}\left(U^{\prime \prime}\right) \text { for all } x \notin U_{i} \Rightarrow U_{i} \bullet \pi\left(U^{\prime \prime}\right) \leq U_{i} \bullet \pi(U) \\
\pi_{y}\left(U^{\prime}\right) & \leq \pi_{y}\left(U^{\prime \prime}\right) \text { for all } y \in U_{i} \Rightarrow U_{i} \bullet \pi\left(U^{\prime}\right) \leq U_{i} \bullet \pi\left(U^{\prime \prime}\right)
\end{aligned}
$$

and strategyproofness follows.

Theorem 3 Assume $|N| \geq 6$ and $|A| \geq 6$. There is no mechanism meeting ex-ante efficiency, preference monotonicity, and positive shares.

\section{Remark 2}

In the statement of Theorem 2, we could replace Preference Monotonicity by the Outcome Monotonicity $(\mathrm{OM})$ property. OM compares two problems $(N, A, U)$ and $\left(N, A^{\prime}, U^{\prime}\right)$ where $A \underset{f}{\subsetneq} A^{\prime}$ and $U$ is the restriction of $U^{\prime}$ to $A$. It requires $\pi_{a}\left(A^{\prime}, U^{\prime}\right) \leq \pi_{a}(A, U)$ for all $a \in A$ : the appearance of new outcomes in the feasible set cannot result in a larger time share for any old outcome.

The interpretations of PM and OM are similar. The two properties are closely related logically as well. See the Appendix for details.

\section{A family of ex-ante efficient solutions}

The simplest way to define ex-ante efficient solutions to a given problem $(N, A, U)$ is to maximize a collective utility function. The additively separable collective utility functions are especially relevant to our set of axioms.

Given an increasing and concave function $f$ defined on $[0,1]$, any lottery $p$ maximizing the separably additive utility function $\sum_{N} f\left(u_{i}\right)=\sum_{N} f\left(U_{i} \bullet p\right)$ is ex-ante efficient. If $f$ is strictly concave, the utility profile of any such lottery is unique for all $U$. We can clearly define an anonymous and neutral selection from the set of optimal lotteries. If $f$ is not strictly concave, so that there may be more than one optimal utility profile, it is again possible to select a solution within the convex optimal set in such a way that the resulting mechanism is anonymous and neutral. The utilitarian solution is an example.

We call an $f$-solution any lottery maximizing the $f$-collective utility, and speak of an $f$-mechanism if an $f$-solution is chosen for any problem.

It turns out that many $f$-solutions meet the fair welfare share. This is true if $f$ takes the form $f(u)=g(\log u)$ where $g$ is increasing and concave (Bogomolnaia et al. [2003]). Two important examples are the Nash solution corresponding to $f(u)=\log u$, and, abusing language, the egalitarian solution corresponding to the limit of $f$-mechanisms where $f$ is increasingly concave, e.g., $f(u)=-|\log u|^{t}$ and $t$ goes to infinity. The latter solution maximizes the leximin ordering (the lexicographic ordering over utility profiles rearranged increasingly).

On the other hand, $f$-mechanisms do not lead to any new example of strategyproof mechanisms. Bogomolnaia et al [2003] show that if an $f$-mechanism is 
strategyproof, the function $f$ must be linear, hence the mechanism is essentially utilitarian.

We conclude with a three outcomes example where we compare the Nash and egalitarian solutions with the two lead solutions of this paper, namely utilitarian and random priority.

Example 2. Let $A=\{a, b, c\}$ and $n$ agents be partitioned in three groups: $n_{x}$ agents like $y, z$ and dislike $x$, where $\{x, y, z\}=A$. We set $\gamma_{x}=\frac{n_{x}}{n}$ and assume $\gamma_{a}>\gamma_{b}>\gamma_{c}>0$.

The two extremist solutions are the utilitarian and egalitarian ones: the latter picks $\pi_{x}=\frac{1}{3}$ for $x=a, b, c$, guaranteeing the utility $\frac{2}{3}$ to every agent; the former chooses $c$ with probability 1 , so that a majority of agents get full utility, whereas the minority gets null utility.

The Nash solution is as follows:

$$
\begin{aligned}
& \frac{1}{2} \geq \gamma_{a} \Rightarrow\left\{p_{x}=1-2 \gamma_{x} \text { and } u_{i}=2 \gamma_{x} \text { if } i \in N_{x}\right\} \text { for all } x=a, b, c \\
& \gamma_{a} \geq \frac{1}{2} \geq \gamma_{b} \Rightarrow\left\{\begin{array}{c}
p_{a}=0, u_{i}=1 \text { if } i \in N_{a} \\
p_{y}=\frac{\gamma_{x}}{\gamma_{b}+\gamma_{c}}=u_{i} \text { for } i \in N_{x} \text { and }\{x, y\}=\{b, c\}
\end{array}\right.
\end{aligned}
$$

Thus a majority of agents gets full utility if and only if they have identical preferences.

The random priority solution offers another compromise between the utilitarian and egalitarian solutions. For all $\{x, y, z\}=\{a, b, c\}$ :

$$
p_{x}=\gamma_{y} \cdot \gamma_{z} \cdot\left(\frac{1}{\gamma_{x}+\gamma_{y}}+\frac{1}{\gamma_{x}+\gamma_{z}}\right) ; u_{i}=\gamma_{x} \cdot \frac{1-\gamma_{y} \cdot \gamma_{z}}{\left(1-\gamma_{y}\right) \cdot\left(1-\gamma_{z}\right)} \text { for } i \in N_{x}
$$

For instance, if $p_{a}=\delta, p_{b}=p_{c}=\frac{1-\delta}{2}$, the corresponding utility profile is

$$
u_{a}=\frac{\delta(3-\delta)}{1+\delta} ; u_{b}=u_{c}=\frac{1}{2}+\frac{(1-\delta)^{2}}{2(1+\delta)}
$$

Here the random priority solution yields a less egalitarian utility profile than the Nash solution for $0 \leq \delta \leq \frac{1}{3}$, a more egalitarian one for $\frac{1}{3} \leq \delta \leq 1$.

\section{References}

[1] Barberà, S., 1979. "Majority and Positional Voting in a Probabilistic Framework," Review of Economic Studies, 46, 379-389.

[2] Barberà, S., A. Bogomolnaia and H. Van Der Stel, 1998. "Strategyproof probabilistic rules for expected utility maximizers," Mathematical Social Sciences, 35, 2, 89-103. 
[3] Bogomolnaia, A. and H. Moulin, 2001. "Random matching under dichotomous preferences," forthcoming, Econometrica.

[4] Bogomolnaia, A., H. Moulin and R. Stong, 2003. "Efficient and fair decision rules under dichotomous preferences," mimeo.

[5] Brams, S. and P. Fishburn, 1978. "Approval voting," American Political Science Review, vol. 72, 3, 831-47.

[6] Dutta, B., H. Peters and A. Sen, 1999. "Strategyproof probabilistic mechanisms in economies with pure public goods," mimeo, Indian Statistical Institute, Delhi.

[7] Freixas, X., 1984. "A cardinal approach to straightforward probabilistic mechanisms," Journal of Economic Theory, vol 34, 227-251.

[8] Gibbard, A., 1977. "Manipulation of Schemes that Mix Voting With Chance," Econometrica, 45, 665-681.

[9] Gibbard, A., 1978. "Straightforwardness of Game Forms with Lotteries as Outcomes," Econometrica, 46, 595-614.

[10] Holzman, R., 2003, private communication.

[11] Hylland, A., 1980. "Strategyproofness of Voting Procedures with Lotteries as Outcomes and Infinite Sets of Strategies," Discussion Paper, Institute of Economics, University of Oslo.

[12] Nandeibam, S., 2001. "The Structure of decision schemes with Von Neumann-Morgenstern preferences," mimeo, University of Birmingham, U.K. 


\section{APPENDIX: Proofs}

\section{Lemma 1}

We fix a problem $(N, A, U)$ and write $p$ for the lottery just defined.

Horizontal equity is clear. To check ex-ante efficiency of $p$, define $N_{2}=\cup_{B} U^{a}$ and consider $W=\frac{1}{m} \sum_{N_{1}} U_{i}+\frac{1}{m-1} \sum_{N_{2}} U_{i}$. By construction $W(a)=1$ if $a$ is in $A_{1}$ or $B$. If $a \notin A_{1},\left|U^{a} \cap N_{1}\right|=m-k$ for some $k, 1 \leq k \leq m$ and $\left|U^{a} \cap N_{2}\right| \leq k-1$, therefore $W(a)<1$. Thus $W(p)>W(q)$ for any lottery $q$ that gives some positive weight to $A \backslash\left(A_{1} \cup B\right)$, and $q$ is not Pareto superior to $p$. Next if the support of $q$ is contained in $A_{1} \cup B$, we have $W(p)=W(q)$ and $U_{i} \cdot q=0$ for all $i \notin N_{1} \cup N_{2}$, therefore $q$ is not Pareto superior to $p$ either.

Now to strategyproofness. We pick an agent $i$, a profile $U$ and a misreport $U_{i}^{*}$ by this agent. We write $A_{1}^{*}, N_{1}^{*}$, etc..., for the characteristics of the problem $\left(N, A, U^{*}\right)$ after this misreport. We assume below $i \in N_{1}$ and analyze the impact of this switch on the partition of $A$ as $A_{1}, B, C=A \backslash\left(A_{1} \cup B\right)$. The easier case $i \notin N_{1}$ is omitted for brevity.

We will need the following finer partition:

$$
\begin{gathered}
X=A_{1} \cap U_{i} \cap U_{i}^{*} ; X^{\prime}=A_{1} \cap U_{i} \cap U_{i}^{* c}, Z=A_{1} \cap U_{i}^{c} \cap U_{i}^{* c} ; Z^{\prime}=A_{1} \cap U_{i}^{c} \cap U_{i}^{*} \\
Y=B \cap U_{i}^{* c} ; Y^{\prime}=B \cap U_{i}^{*}\left(\text { recall } B \subseteq U_{i}^{c}\right)
\end{gathered}
$$

The new partition $A_{1}^{*}, B^{*}, C^{*}$ depends much on whether $m^{*}$ equals $m, m-1$ or $m+1$. The case $m^{*}=m$ is the most involved, so we explain it in detail, leaving the other two similar cases to the reader.

Observe that $Z^{\prime}=\varnothing$, and $X \cup Z \neq \varnothing$. It is easy to check that $A_{1}^{*}=$ $X \cup Z \cup Y^{\prime} \cup D$, where $D \subseteq C \cap U_{i}^{c} \cap U_{i}^{*}$, whereas $B^{*} \subseteq X^{\prime} \cup Y \cup C$. Define $\widehat{X}=B^{*} \cap X^{\prime}, \widehat{Y}=B^{*} \cap Y$ and $\widehat{C}=B^{*} \cap C$. We prove now the two following properties

$$
\begin{gathered}
\widehat{Y} \underset{\neq}{\subset} \Longrightarrow Y^{\prime} \cup D \neq \varnothing \\
\widehat{C} \cap U_{i} \neq \varnothing \Longrightarrow X=\varnothing
\end{gathered}
$$

Indeed if $a \in B^{*} \cap C$, then $U^{* a}=m-1$, so $a \in U_{i} \cap U_{i}^{* c}$ would imply $U^{a}=m$, contradicting $a \in C$. Thus $a \in \widehat{C} \cap U_{i}$ implies $a \in U_{i}^{*}$, and by definition of $B^{*}$ we must have $U_{i}^{*} \cap A_{1}^{*}=\varnothing$ in particular $X=\varnothing$.

To prove the former claim, pick $a \in Y \backslash \widehat{Y}$, and note that $a \in U_{i}^{c} \cap U_{i}^{* c}$, so that $U^{a}=U^{* a}$ : the support of $a$ is unchanged in the switch from $U_{i}$ to $U_{i}^{*}$. Therefore $a \notin B^{*}$ is possible only if $A_{1}^{*}$ contains outcomes outside $A_{1}$, namely $Y^{\prime} \cup D$ is nonempty.

We compute now 


$$
U_{i} \bullet p^{*}=\frac{w X+\widehat{X}+\left(\widehat{C} \cap U_{i}\right)}{w\left(X+Z+Y^{\prime}+D\right)+\widehat{X}+\widehat{Y}+\widehat{C}}
$$

By $\widehat{C} \cap U_{i} \leq \widehat{C}$ and $\widehat{X} \leq X^{\prime}$, we get

$$
U_{i} \bullet p^{*} \leq \frac{w X+X^{\prime}+\left(\widehat{C} \cap U_{i}\right)}{w\left(X+Z+Y^{\prime}+D\right)+X^{\prime}+\widehat{Y}+\left(\widehat{C} \cap U_{i}\right)}
$$

From our first observation and $Y+Y^{\prime} \leq A-1 \leq w$, we have

$$
w\left(Y^{\prime}+D\right)+\widehat{Y} \geq Y^{\prime}+Y=B
$$

Therefore $U_{i} \bullet p^{*} \leq U_{i} \bullet p$ reduces to

$$
\frac{w X+X^{\prime}+\left(\widehat{C} \cap U_{i}\right)}{w(X+Z)+X^{\prime}+B+\left(\widehat{C} \cap U_{i}\right)} \leq \frac{w\left(X+X^{\prime}\right)}{w\left(X+X^{\prime}+Z\right)+B}=U_{i} \bullet p
$$

If $X>0$, we have $\widehat{C} \cap U_{i}=0$ (second observation above). Then it is enough to take $B=0$, and we are done. If $X=0$, recall $Z>0$, and we also have $X^{\prime}>0$. Setting $E=\widehat{C} \cap U_{i}$, we have now $w X^{\prime} \geq A-1 \geq X^{\prime}+E$. Therefore it is enough to show the inequality above for $B=0$. It boils down to $w X^{\prime} \geq X^{\prime}+E$ and the proof is complete.

\section{Proposition 2}

We assume $N=\{1,2,3\}$ and $A=\{a, b, c\}$. The generalization to more agents or more outcomes is clear, by "neutralizing" all but three agents and outcomes (i.e., neutral agents like no outcome and neutral outcomes are liked by no one). We fix an ex-post efficient mechanism meeting GSP and derive a contradiction.

Let, when agent 1 likes only $a$, agent 2 likes only $b$, and agent 3 likes only $c$, the solution be $p=\left(p_{1}, p_{2}, p_{3}\right)$. Without loss of generality we can suppose that $p_{1}>0$. We now fix preferences of agents 2 and 3 , and consider changes in preferences of agent 1 only.

When agent 1 likes all three outcomes, EXP implies that $a$ gets zero probability and hence the solution is $q=\left(0, q_{2}, q_{3}\right)$, where $q_{2}+q_{3}=1$. Note that when agent 1 likes all outcomes she always gets utility 1 . Therefore she can deny liking some outcomes without decreasing her utility. GSP implies then that such a lie by agent 1 cannot benefit any other agent. Hence, if agent 1 announces to like $a$ and one of $b, c$ and the preferences of agents 2 and 3 remain fixed, the solution must still be $q=\left(0, q_{2}, q_{3}\right)$.

Thus, if agent 1 likes $\{a, b\}$, then she gets $1-q_{3}$. However in this case, if she lies and denies $b$, she receives utility $1-p_{3}$. Hence, strategyproofness requires $p_{3} \geq q_{3}$. Analogously, we obtain $p_{2} \geq q_{2}$. Therefore, $p_{2}+p_{3} \geq q_{2}+q_{3}=1$, and so $p_{1}=0$, a desired contradiction.

3. Proposition 3 
We assume $|A|=6, N=\{1,2,3,4\}$, and fix a mechanism satisfying the four properties listed in the statement. We use the following notations: an outcome $a$ is identified (by neutrality) with the coalition of agents who like $a$, and a profile is written as a list of at most six coalitions, with the understanding that the list is completed by empty coalitions.

We compute the lotteries selected at a handful of simple profiles, and write each statement as $[12,34] \rightarrow\left(\frac{1}{2}, \frac{1}{2}\right)$, where the right-hand side lists the probabilities of all the efficient outcomes in the order in which they appear in the profile on the left-hand side. The above statement follows from anonymity.

Consider $[12,34,3,3,4] \rightarrow(p, 1-p)$, where the shares are for the two efficient outcomes 12 and 34 . Notice that $p<\frac{1}{2}$ allows a profitable misreport by coalition $\{3,4\}$ at $[12,34]$, whereas $p>\frac{1}{2}$ allows such a move by $\{3,4\}$ at $[12,34,3,3,4]$. Therefore $[12,34,3,3,4] \rightarrow\left(\frac{1}{2}, \frac{1}{2}\right)$. Next, agent 3 denying alternative "34" at this profile leads to the profile $[12,3,3,4,4] \rightarrow(z, t, t, t, t)$ (by anonymity and neutrality). By strategyproofness we obtain that $t \leq \frac{1}{6}$ and so $z+t \geq \frac{1}{2}$.

Further, anonymity and neutrality give $[12,23,13,14,24,34] \rightarrow\left(\frac{1}{6}, \frac{1}{6}, \frac{1}{6}, \frac{1}{6}, \frac{1}{6}, \frac{1}{6}\right)$ and $[12,23,13,4,4,4] \rightarrow(u, u, u, v, v, v)$. Joint misreport at the first of these profiles by the coalition $\{1,2,3\}$, denying the alternatives liked by agent 4 , leads to the second one, and would give each its member $2 u+v$. Weak GSP implies then $2 u+v \leq \frac{1}{2}$, and hence $v \geq \frac{1}{6}$. Compare now the second profile with $[12,23,13,4,4] \rightarrow(x, x, x, y, y)$. If at this last profile agent 4 pretends to like the remaining 6 -th alternative, she gets $2 v$. By strategyproofness we have that $y \geq v \geq \frac{1}{6}$ and so $x \leq \frac{2}{9}$.

Finally, assume that at $[12,23,13,4,4]$ agents 1 and 2 deny alternatives agent 3 likes. It results in the profile $[12,3,3,4,4]$ and each of 1,2 gets $z+t \geq \frac{1}{2}$, while by annnoncing their true preferences they would only get $2 x \leq \frac{4}{9}$ each. This last observation contradicts weak GSP.

\section{Theorem 1}

Assume to the contrary that there exists a mechanism $\pi$, satisfying the premises of the theorem. We will need the following two lemmas.

Lemma 3 Suppose that $p=\pi(U), p_{a}=0$, and an agent $i$ likes the outcome $a$. If $i$ changes her message by denying liking a, her utility remains unchanged and the outcome a still gets zero probability.

Proof. Let $u_{i}$ and $u_{i}^{\prime}$ be agent's $i$ utility, respectively under her true and falsified preferences, and $p_{a}^{\prime}$ be the probability of $a$ under the latter. SP at the two corresponding profiles $U, U^{\prime}$ implies $u_{i} \geq p_{a}^{\prime}+u_{i}^{\prime} \geq p_{a}^{\prime}+\left(u_{i}-p_{a}\right)=p_{a}^{\prime}+u_{i}$. Hence, $u_{i}=u_{i}^{\prime}$ and $p_{a}^{\prime}=0$.

Lemma 4 Suppose that an outcome $a$ is the only one liked by all agents from $M, U^{a}=M=\left\{i_{1}, \ldots, i_{m}\right\}$, for any $i_{k} \in M$ there exists an outcome $b_{k}$ liked by $i_{k}$ only, and there is no outcome liked by some agents from $M$ and some from $N-M$. Then $p_{a} \geq m / n$. 
Proof. We proceed by induction on $m$. The case $m=1$ is a special case of FS. Assume $m>1$. Note that EXP implies $u_{i}=p_{a}$ for all $i \in M$.

Suppose that there is no outcome liked by all agents in $M-i_{m}$. If agent $i_{m}$ claims to only like outcome $b_{m}$ (with resulting solution $p^{\prime}$ ), then by FS $p_{b_{m}}^{\prime} \geq 1 / n$, and by induction $p_{a}^{\prime} \geq(m-1) / n$. Hence by SP, $p_{a}=u_{i_{m}} \geq$ $p_{b_{m}}^{\prime}+p_{a}^{\prime} \geq m / n$.

Suppose now that there are some outcomes liked by all agents in $M-i_{m}$. By EXP they all must get zero probability. Hence by the previous lemma agent $i_{1}$ can lie and deny liking those outcomes without changing her utility $u_{i_{1}}=p_{a}$. The above argument applied to the new preference profile gives $p_{a} \geq m / n$ again.

From here on assume $N=\{i, j, k, l, m\}$. For any outcome $\alpha$ call the set $U^{\alpha}$ a "coalition". By EXP, only outcomes liked by inclusion maximal coalitions receive positive probability. We will concentrate on the preference profiles under which there is an outcome $\alpha$, such that $U^{\alpha}=\{i, j, k\}$, and all other coalitions are of size at most 2 . We will represent such profiles graphically, with vertices for the agents, a shaded triangle to denote outcome $\alpha$ and edges to denote maximal coalitions of size 2. The six instances of interest are shown on Figure 5.

We will assume that all non-empty non-maximal coalitions are singletons, i.e. coming from outcomes liked by only one agent. We will further insist that each agent likes exactly 4 outcomes. Thus each diagram on the Figure 5 fully specifies the non-maximal coalitions as well. In the argument below we will often imagine an agent lying, claiming to like or dislike a certain outcome. In each of those cases we will also assume the agent who lies to keep a total of 4 outcomes of which she likes, either by denying one of the outcomes only she likes or claiming to like an outcome no one likes. We will not explicitly specify these fixes below. Our assumption $|A| \geq 17$ allows in each case to construct a preference profile with the above features. For instance for the profile corresponding to the case $\mathrm{B}$ we need 11 singleton-liked outcomes in addition to the 4 outcomes liked by at least two agents; two of the remaining "empty" outcomes will be used when we consider below a possibility that the agent 1 denies outcomes $[1,13]$ and $[1,3]$ (and thus implicitly assume he claims to like instead another two outcomes, previously not liked by anybody).

In Figure 5 each maximal coalition is labelled by the probability it receives under our mechanism $\pi$. We will derive relations between those probabilities below. Note that we have used anonymity and neutrality assumptions to label certain edges with the same variable. To reference certain agents we have labelled some vertices by circled positive integers. We will denote the outcome liked by coalition $(x, y)$ as $[x, y]$.

Note also that certain edges were labelled by zero. This follows from neutrality and EXA as follows. Consider case F. By neutrality the four outcomes $[9,11],[9,12],[10,11]$, and $[10,12]$ must all have the same probability. However if $[9,11]$, and $[10,12]$ have positive probability, then we can obtain a Pareto superior solution by splitting this probability equally between the outcomes $\alpha$ and $[11,12]$. A similar remark gives zeroes in case $\mathrm{D}$ and shows that in case $\mathrm{E}$ either $i$ or $k$ is zero. 
Since certain edges have zero probability, we can now apply Lemma 3.

Looking at 10 denying $[10,11]$, we see $m=h+k$.

Looking at 11 denying $[10,11]$, we see $1-m=1-h-j-k$, or $m=h+j+k$, hence $j=0$.

Looking at 10 denying $[10,11]$ and $[10,12]$, we see $m=b$.

Looking at 12 denying $[9,12]$ and $[10,12]$, we see $1-m=1-e-2 f$, or $m=e+2 f$.

Looking at 7 denying $[7,8]$, we see $g=h+i($ since $j=0)$.

Now suppose 3 denies $[1,3]$ and simultaneously claims to approve an outcome $[2,3]$. In this case 3 gets $1-g$, hence by SP we must have $1-b-c \geq 1-g$ or $g \geq b+c$. Next suppose 4 denies $[4,5]$ and simultaneously claims to approve an outcome $[4,6]$. In this case 4 gets $g$, hence by SP we must have $e+f \geq g$. Combining these we see that $e+f \geq h+i=g \geq b+c \geq b=m=h+k=e+2 f$.

Thus $c=f=0$ and $i=k$, but since either $i$ or $k$ must be zero, this says $i=k=0$. Hence $b=e=g=h=m$. Applying Lemma 1 to 1 denying $[1,13]$ and $[1,3]$, we see $a=b$. Applying Lemma 2 to case A twice we see that $a \geq 3 / 5$ and $1-a \geq 2 / 5$, hence $a=b=e=g=h=m=3 / 5$.

Consider now what happens if the true preferences are given by case $\mathrm{B}$ from Figure 5. An agent 1 will have utility $b=2 / 5$. Suppose he lies, denying the outcome $\alpha$, liked by three agents coalition. The resulting profile is shown on Figure 6 . By Lemma $4, \alpha$ will receive at least $2 / 5$ and by neutrality the remaining outcomes liked by maximal coalitions of size 2 will each receive a third of whatever is left, i.e. at most $1 / 5$. But that means that agent 1 will have utility at least $4 / 5$. Thus SP is violated and no proposed mechanism exists.

\section{Theorem 2}

Suppose we have a mechanism meeting PM, EXA and PS. We will look for outcomes that get zero probability. By PM if an outcome gets zero probability and we increase the popularity of any other outcomes, then that outcome still gets zero probability. We will use this remark to find an instance in which all outcomes approved by a particular agent get zero probability, contradicting PS.

Consider 6 agents $\{0,1,2,3,4,5\}$. Suppose $(i, j, k, m)$ is an (ordered) quadruple of distinct elements of $\{1,2,3,4,5\}$. We will first look at sets of 4 outcomes. These four outcomes will be liked respectively by coalitions $\{i, j\},\{j, k\},\{k, m\}$, and $\{m, i\}$ (forming a "square"). Suppose we add that agent 0 likes one of these four outcomes. For example, if we add agent 0 liking $\{m, i\}$, then we obtain coalitions $\{i, j\},\{j, k\},\{k, m\}$, and $\{0, m, i\}$. For this example EXA requires either $\{i, j\}$ or $\{k, m\}$ to get zero probability. If $\{i, j\}$ gets zero probability then we will say $\{m, i\}$ zeroes $\{i, j\}$ and write $\{m, i\} \longrightarrow\{i, j\}$. Note that each edge of the square zeroes at least one of its "neighbors".

Suppose $\{m, i\} \longrightarrow\{i, j\}$ and $\{k, m\} \longrightarrow\{j, k\}$ Then it follows from PM that if we add agent 0 liking both $\{m, i\}$ and $\{k, m\}$ (resulting in coalitions $\{i, j\},\{j, k\},\{0, k, m\}$, and $\{0, m, i\})$ both $\{i, j\}$ and $\{j, k\}$ get zero probability and hence $u_{j}=0$, contradicting PS. Thus the square must be "oriented", either $\{m, i\} \longrightarrow\{i, j\} \longrightarrow\{j, k\} \longrightarrow\{k, m\} \longrightarrow\{m, i\}$, or the reverse. In particular, each edge zeroes only one of its neighbors. 
Consider 6 outcomes with respective coalitions $\{1,2\},\{2,3\},\{3,4\},\{4,1\}$, $\{1,5\}$, and $\{5,3\}$. These 6 outcomes form 3 squares $(1,2,3,4),(1,2,3,5)$, and $(1,5,3,4)$. It is impossible to orient all 3 squares in such a way that the orientations of any two squares coincide on their two common ages. Therefore without loss of generality we may assume $(1,2,3,4)$ is oriented $\{4,1\} \longrightarrow\{1,2\} \longrightarrow$ $\{2,3\} \longrightarrow\{3,4\} \longrightarrow\{4,1\}$ and $(1,2,3,5)$ is oriented $\{1,5\} \longrightarrow\{5,3\} \longrightarrow$ $\{3,2\} \longrightarrow\{2,1\} \longrightarrow\{1,5\}$. Suppose we add that agent 0 likes outcomes $\{1,4\}$ and $\{3,5\}$. From the square $(1,2,3,4)$ and 0 liking $\{1,4\}$ we see $\{1,2\}$ gets zero probability. From the square $(1,2,3,5)$ and 0 liking $\{3,5\}$ we see that $\{2,3\}$ gets zero probability. Therefore $u_{2}=0$ contradicting PS.

\section{Remark 2}

The above proof can be easily adapted to show that if $|N| \geq 6$, then there is no mechanism meeting ex-ante efficiency, Outcome Monotonicity, and Positive Shares. Rather than adding the fact that agent 0 likes an outcome $\{m, i\}$ we add a new outcome liked by the coalition $\{0, m, i\}$.

We note that the two properties PM and OM are closely related. First, if we make the assumption that adding "empty" outcomes does not change the solution, then PM clearly implies OM. Conversely, if the mechanism is neutral and EXP, then OM implies PM.

Indeed, suppose an agent $i$ does not like an outcome $a$. Add a new outcome $b$, such that $U^{b}=U^{a} \cup\{i\}$. By OM, the probabilities for all outcomes except $b$ do not increase. By EXP outcome $a$ now gets zero probability. Again by OM, deleting outcome $a$ will not decrease the probabilities of other outcomes. Since these probabilities sum to one, deleting $a$ will not change the probabilities. Thus we can replace $a$ by $b$, which is equivalent to changing agent's $i$ preferences in favor of $a$, and the probability of other outcomes will not increase. By neutrality outcomes are interchangeable, and PM follows.

\begin{tabular}{|c|c|c|c|c|}
\hline$a$ & $b$ & $c$ & $d$ & $e$ \\
\hline 1 & & 1 & & \\
\hline 1 & & & 1 & \\
\hline 1 & & & & 1 \\
\hline & 1 & 1 & & \\
\hline & 1 & & 1 & \\
\hline & 1 & & & 1 \\
\hline
\end{tabular}

Figure 1

\begin{tabular}{|c|c|c|}
\hline$a$ & $b$ & $c$ \\
\hline 1 & & \\
\hline & 1 & \\
\hline 1 & 1 & \\
\hline
\end{tabular}

Figure 2 


\begin{tabular}{|c|c|c|}
\hline$a$ & $b$ & $c$ \\
\hline 1 & & \\
\hline 1 & 1 & \\
\hline & 1 & 1 \\
\hline & & 1 \\
\hline
\end{tabular}

Figure 3

\begin{tabular}{|l|c|c|c|c|c|c|}
\hline & $a$ & $b$ & $c$ & $d$ & $e$ & $f$ \\
\hline 1,2 & 1 & 1 & 1 & 1 & & \\
\hline 3 & 1 & & 1 & & & \\
\hline 4 & 1 & 1 & & 1 & & \\
\hline 5,6 & 1 & 1 & & & & \\
\hline 7 & & 1 & & & & \\
\hline 8,9 & & & 1 & 1 & 1 & \\
\hline 10,11 & & & & & 1 & \\
\hline $12,13,14$ & & & & & & 1 \\
\hline
\end{tabular}

Figure 4 
24

Figure 1: 